\title{
CONTROLE DE PLANT AS DANINHAS COM CYANAZINE APLICADO EM MISTURA COM OUTROS HERBICIDAS, NA CULTURA DO ALGODÃO (Gossypium hirsutum L,)
}

\section{JULIO PEDRO LACA-BUENDIA}

Pesq uisad or/EPAMIG

Av. Amazonas, 115 - Sala 60930.180

- Belo Horizonte, MG

\section{RESUMO}

Com a finalidade de estudar a mistura de tanque mais eficiente com cyanazine em aplicação de pré-emergência na cultura algodoeira (Gossypium hirsutum L.), foram estudados os seguintes tratamentos: cyanazine + diuron nas doses de $0,8+0,8 \mathrm{~kg}$ i.a/ha e $1,0+1,0 \mathrm{~kg}$ i.a/ha; cyanazine+ oryzalin, nas do sés de $1,2+0,8 \mathrm{~kg}$ i.a/ha e $1,6+1,2 \mathrm{~kg}$ i.a/ha; cyanazyne + metola chlor, nas doses de $1,4+2,0 \mathrm{~kg}$ i.a/ha e 1,75 $+2,52 \mathrm{~kg}$ i.a/ha;cia nazine na dose de 1,75 $\mathrm{kg}$ i.a /ha; oryzalin na dose de $1,12 \mathrm{~kg}$ i.a/ha; metolachlor na dose de $2,52 \mathrm{~kg}$ i.a /ha e diuron na dose de $1,6 \mathrm{~kg}$ i.a /ha. Para efeito de comparação, utilizou-se uma testemunha sem capina e outra com capi na manual.

Nenhum tratamento apresentou injúria para as plantas de algodão e não houve diferenças significativas para o "stand" inicial. Já no "stand" final, a teste munha sem capina apresentou o menor número de plantas, sendo que não houve diferenças significativas dos outros tratamentos com a test emunh a capi nada.

Para o rendimento, a mistu ra cyanazine + metolachior em ambas as doses estudadas, não apre- sentaram diferenças significativas da test emunh a capinada.

Quan to à altura da planta, peso de 100 sementes, porcentagem e índice de fibras não houve diferenças significativas entre os tratamentos estudados, somente o peso do capulho foi afetado pelo oryzalin.

Pela avaliação visual (EWRC 1 a 9)*, os herbicidas apresentaram um controle satisfatório somente até os 30 dias após aplicação, sendo que a mistura cyanazine + metolachlor foi eficiente quanto a test emunh a capinada.

No controle da Portulaca oleracea, a mistura cyanazine + ory zalin na maior dose e oryzalin apresentaram $71,4 \%$ de controle ate os 30 dias e $79,4 \%$ e $82,4 \%$, respectivamente, até 45 dias da aplicação. Para Amaranthus sp., à exceção da cyanazine e cyanazine + diuron nas doses menores, não apresentaram nenhum controle, sendo que os outros herbicidas controlar am com eficiência superior a $70 \%$. Para Centratherium punctatum, o cyanazine apresent ou 78,2\% e 73,4\%, respectivamente, após 50 e 45 dias da aplicação. Para Cyperus

* European Weed Research Council. 
sp e \& Brachiaria plantaginea, o metolachlor sozinho ou em mistura com cyanazine, apresentou uma eficiência de 90\% para Cyperus sp. e de $70 \%$ para $B$. plan tagi nea até 45 dias da apli cação. Para as espécies não dominantes (maioria dicotiledôneas), o melhor controle foi de cyanazine + metolachlor na dose maior, com $70,5 \%$ e $60,2 \%$, respectivamente, após 30 e 45 dias da aplicação. Para o total das espécies, cyanazine + metol achlor, em ambas doses estudadas, apres entaram controle de $66,2 \%$ e $67,3 \%$, respectivamente, após 30 dias e $63,3 \%$ e $64,3 \%$, respectivamente, após 45 dias da aplic ação.

Para as análises tecnológicas da fibra, não houve diferenças significativas na maturação da fibra, uniformidade de comprimento, índice Macronaire e índice Pressley. No comprimento da fibra, a mistura de cyanazine $+\operatorname{diuron}(0,8+0,8 \mathrm{~kg}$ $\mathrm{ia} / \mathrm{ha})$, apresentou o maior comprimento $(26,1 \mathrm{~mm})$ e cyanazine+ metolachlor $(1,75$ $+2,52 \mathrm{~kg}$ i.a / ha), o menor comprimento $(24,9 \mathrm{~mm})$, sem diferenças significativas com as teste munhas.

PALA VRAS-CHAVE: Algodão herbáceo Gossypium hirsutum L., controle de plantas daninhas, mistu ra de herbicidas.

\section{SUMMARY}

WEED CONTROL IN COTTON (Gossypium hirsutum L.) WITH CYANAZINE AND OTHER HERBICIDES

The objective of this study was to determine the most efficient combination of cyanazine with other herbicides as preemergent applications in cotton (Gossypium hirsu- tum L.). The following treatments were tested: cyanazine + diuron at $0.8+0.8 \mathrm{~kg}$ i.a/ha and $1.0+1.0 \mathrm{~kg}$ i.a/ha; cyanazine + oryzalin at $1.2+0.8 \mathrm{~kg}$ i.a / ha and $1.6+1.2$ $\mathrm{kg}$ i.a/ha; cyanazine + metolachlor at $1.4+$ $2.0 \mathrm{~kg}$ i.a/ha and $1.75+2.52 \mathrm{~kg}$ i.a/ha; cyan azine at $1.75 \mathrm{~kg}$ i.a/ha; ory zalin at 1.12 $\mathrm{kg}$ i.a/ha; metolachlor at $2.52 \mathrm{~kg}$ í.a/ha; diuron at $1.6 \mathrm{~kg}$ i.a/ha; unweeded and weed - free checks. Based on visual eva luation according to European Weed Research Council scale, all the herbicides showed good weed control up to 30 days - after application (d.a.a). None of the treatments caused injuries to cotton plants and no significant difference was observed for initial and final stand. Cyanazine + metolachlor did not show any significant difference for seed - control yield as compared to weed - free check. Plant height, seed index, fiber percentage, lint index and fiber properties were not affected by any of the treatments. The cotton boll weight was affected by oryzalin.

KEYWORDS: Herbaceous cotton, Goss ypiu m hirsutum L., weed control, herbicide mixtures.

\section{INTRO DUÇÃO}

Para um desenvolvimento normal, o algodoeiro exige solos livres das plantas daninhas que, além da produção, também prejudicam o tipo e as características tecnológicas da fibra. Dependendo da espécie e do tipo de planta daninha, bem como do período crítico de sua concorrên cia, o rendimento do algodão pode ser redu zido até $90 \%(8)$. 
Num programa de aplicação de herbicidas, o período critico de competição deve ser levado em con ta na escolha de um herbicida, pois relacionara aqueles que possuem um ef eito residual apropriado, ou seja, de pelos menos, 45 dias (4 e 7) .

Vários estudos têm mostrado que misturas e combinações de herbicidas apresentam uma maior eficiên cia de controle para um maior número de espécie de plantas daninhas, torn ando-se mais econômico pelo uso de doses menores $(1,2,3,5,6,9,10,11$, 12 e 13).

Este trabalho teve como objetivo, determinar a mistura mais eficiente com cyanazine, aplicada em pré-emergência, para o controle das plantas daninhas, possíveis sintomas de intoxicação às plantas de algodão, efeitos aos componentes agronômicos da cultura e nas características tecnológicas da fibra.

\section{MATE RIAL E MÉTODOS}

Este ensaio foi instalado no Campo Experimental do Gorutuba, no Município de Porteirinha, Norte de Minas, num solo aluvial, franco - siltoso, cujas analise físico-química encontra-se no Quadro 1.

O delineamento experimental usado foi o de blocos casualizados, com doze tratamentos, repetidos quatro vezes. As parcelas eram constituídas de quatro linhas de 5,0 m de comprimento cada, espaçadas de $0,8 \mathrm{~m}$, perfazendo uma área de $16 \mathrm{~m} 2$, sendo a área útil de $8 \mathrm{~m}^{2}$.

A semeadura foi realizada no dia 26/11/80. As sementes foram colocad as de maneira seguida no sulco, deixando-se cair 30 sementes por metro linear. A germinação ocorre u após 9 dias da semeadura. 0 desbaste foi realizado após 23 dias da semeadura. Utilizou-se o cultivar 'Minas Sertaneja'. A adubação foi feita no momento da semeadura, aplicando-se $200 \mathrm{~kg} / \mathrm{ha}$ da fórmula 4:28(30):16 de NPK. Aos 30 dias após a emergência foi realiza da uma adubação em cobertura com $100 \mathrm{~kg} / \mathrm{ha}$ de sulfato de amônio, colocado $0,15 \mathrm{~m}$ ao lado das plantas.

Os tratamentos utilizados foram: cyanazine $^{1 /}+$ diuron ! nas doses de $0,8+0,8$ $\mathrm{kg}$ i.a/ha e $1,0+1,0 \mathrm{~kg}$ i.a/ha; cyanazine + oryzalin $^{3} /$ nas doses de $1,2+0,8 \mathrm{~kg}$ i.a/ha e $1,6+1,2 \mathrm{~kg}$ i.a/ha; cyanazine + metolachlor 4 / nas doses de $1,4+2,0 \mathrm{~kg}$ i.a/ha e 1,75+2,5 $\mathrm{kg}$ i.a/ha; cyanazine na dose de $1,75 \mathrm{~kg}$ i.a/ha; oryzalin na dose de $1,12 \mathrm{~kg}$ ía/ha; metolachlor na dose de $2,52 \mathrm{~kg}$ do i.a/ha e diuron na dose de $1,6 \mathrm{~kg}$ i.a/ha. Para

1/ Bladex 50 SC; 2/ Karmex 80 PM; 3/ Surflan 75 PM; 4/ Dual 72 CE.

Quadro 1. Análise físico -química do solo onde foi instalado o ensaio.

\begin{tabular}{|c|c|c|c|c|c|c|c|c|c|c|c|}
\hline $\begin{array}{c}\text { Areia } \\
\text { grossa } \\
(\%)\end{array}$ & $\begin{array}{l}\text { Areia } \\
\text { Fina } \\
(\%)\end{array}$ & $\begin{array}{c}\text { Silte } \\
(\%)\end{array}$ & $\begin{array}{l}\text { Argi- } \\
1 \mathrm{a}(\%)\end{array}$ & $\begin{array}{c}\mathrm{pH} \\
\text { em } \\
\text { àgua }\end{array}$ & $\begin{array}{l}\mathrm{Al}^{3+} \\
\text { eqmg/ } \\
100 \mathrm{cc}\end{array}$ & $\begin{array}{l}\mathrm{Ca}^{2+} \\
\text { eqmg/ } \\
100 \mathrm{cc}\end{array}$ & $\begin{array}{l}\mathrm{Mg}^{2+} \\
\text { eqmg/ } \\
100 \mathrm{cc}\end{array}$ & $\begin{array}{l}\mathrm{K}^{+} \\
\mathrm{ppm}\end{array}$ & $\begin{array}{c}\mathrm{P} \\
\mathrm{ppm}\end{array}$ & $\begin{array}{l}\text { M.O. } \\
(\% 9\end{array}$ & $\begin{array}{c}\mathrm{N} \\
(\%)\end{array}$ \\
\hline 8 & 24 & 54 & 54 & 6,0 & 0,0 & 4,88 & 1,72 & 135 & 2 & 1,75 & 0,10 \\
\hline
\end{tabular}


efeito de comparação, utilizou-se um tratamento sem capina e outro com capina manual.

A aplicação dos herbicidas foi realizada com pulverizador costal pressurizado com $\mathrm{CO} 2$, à pressão constante de $2,75 \mathrm{~kg} / \mathrm{cm}^{2}$, bico Teejet 110.02, malha 50, a $0,50 \mathrm{~m}$ de altura do solo e o consumo de calda foi de 328 litros/hectare. O solo encontrava-se úmido, após chuva de $3,1 \mathrm{~mm}$, sendo feita a aplicação entre 7:40 a 8:55 horas do dia 27/11/1980, com $24,7^{\circ} \mathrm{C}$ de temperatura do ar as 9 horas.

Para o levantamento das plantas daninhas, que ocorreram em cada tratamento, foram feitas duas contagens, aos 30 e 43 dias após a aplicação, das espécies das plantas daninhas numa área de $0,25 \mathrm{~m}^{2}$,

em quatro locais diferentes da área útil de cada parcela, perfazendo uma área total de $1 \mathrm{~m}^{2}$.

As precipitações pluviais, em milímetros, durante o ciclo da cultura, estão relacionados no Quadro 2.

Foram feitas duas colheitas,sendo a primeira aos 116 dias após a semeadura e a segunda aos 193 dias após a semeadura.

\section{RESULTADOS E DISCUSSÃO}

Os resultados da análise de variância para o "stand" inicial e final, produção, alt ura da planta, peso de capulho, peso de 100 sementes, porcentagem de fibra e índice de fibra, encontram -se no Quadro 3.
No "stand" inicial, após 15 dias da emergência, observou-se que nenhum tratamento apresentou injúrias para as plantas de algodão 'e não houve diferenças significativas entre os tratamentos. Já no "stand" final, houve diferenças significativas, devido a competição de plantas daninhas, sendo que a testemunha apresentou o menor "stand", e os outros tratamentos não se diferenciaram da tes temunha capinada.

$\mathrm{Na}$ produção a mistura de cyanazine + metolachlor, nas doses de 1,4 +2,0 kg i.a/ha e $1,75+2,52 \mathrm{~kg}$ i.a/ha não diferenciou da testemunha capinada (1463 kg/ha) alcançando uma produção de $776 \mathrm{~kg} / \mathrm{h}$ a e 789 $\mathrm{kg} / \mathrm{ha}$, res pectivamente.

Quan to à altura da planta, peso de 100 sementes, percentagem e índice de fibras não houve diferenças significativas entre os tratamentos estudados. 0Opeso do capulho foi afe tado pela aplicação de oryzali n, que não apresentou diferenças significativas com a testemu nha sem capina. Parece que este herbicida, em doses maiores $(1,12 \mathrm{~kg}$ i.a/ha), tem efeitos nega tivos para o peso do capulho, apresentando $3,8 \mathrm{~g}$, devido ao baixo controle das plantas dani nhas.

$\mathrm{Na}$ avaliação visual (EWRC 1-9), feita após 30 e 45 dias da aplicação, verificou-se que os herbicidas apresentaram um con trole satisfatório até " os 30 dias, sen do que a mistura de cyanazine +

Quadro 2. Precipitações pluviais durante o ciclo da cultura em Porteiri nha (MG). Sa fra 1980/1981.

\begin{tabular}{llllllll} 
Nov. & Dez. & Jan. & Fev. & Mar. & Abr. & Mai. & Jun. \\
\hline 96,4 & 317,0 & 117,5 & 3,4 & 362,1 & 37,2 & 7,5 & 941,1 \\
\hline
\end{tabular}



Quadro 3. Resultados médios obtidos no teste de herbicidas isolados ou em misturas de tanque, em
pré-emergência, na cultura algodoeira em Porteirinha, Norte de Minas. Ano Agricola $1980 / 81$.

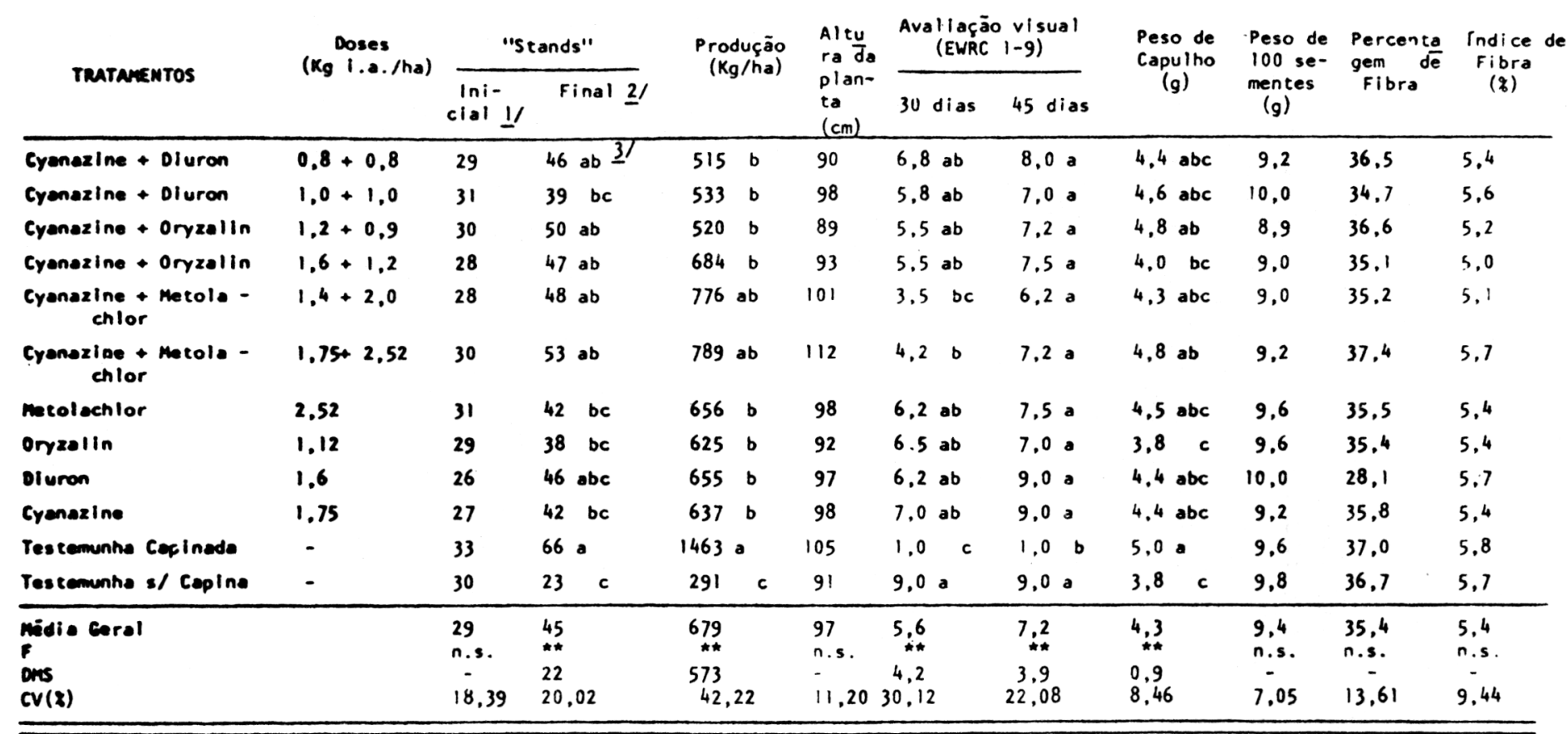

I/ Nümero de plontas em l m linear de área ütil (apös 10 dias da emergência)

2/ Nümero de plantes nas duas fileiras centrais da parcela (na colheita).

3/ médias seguidas pela mesma letra, na mesma coluna, näo diferem significativamente entre si, ao nivel de $5 \%$ de probabilidade pelo teste de Tukey. 
metolachlor na menor dose, mostrou se tão eficiente quanto a testemunha capinada. Os herbicidas apresentaram um baixo poder residual, devido, pos sivelmente, à ins uficiente precipitação ocorrida após sua aplicação.

Quanto ao controle das plantas daninhas, pode-se verificar no Quadro 4, que Portulaca oleracea L. (beldroega), Amaranthus sp. (caruru) , Centratheríum punc tatum (perpétua), Cyperus sp. (tiririca) e Brachiaria plantaginea (Link) Hitch (capim-marmela da) for am as que ocor reram em maior densidade. Observou-se que para beldroega, o melh or controle foi obtido com a mist ura de cyanazine + oryzalin, na dose de $1,6+1,2 \mathrm{~kg}$ i.a/ha e oryzalin, na dose de $1,12 \mathrm{~kg}$ i.a /ha, com $71,4 \%$ de controle, respectivamente, após 30 dias da aplicação e $79,4 \%$ e $82,4 \%$, res pectivamente, a pós 45 dias da aplicação. o caruru somente não foi cont rolado pelo ory zal in, na dose de $1,12 \mathrm{~kg}$ i.a/ha e cyanazine e diuron, na dose de $0,8+$ $0,8 \mathrm{~kg}$ i.a/ha, sendo que todos os outros tratamentos o controlaram com eficiência superior a 70\%. Para perpétua, o melh or controle foi apresentado pelo cyanazi ne na dose de $1,75 \mathrm{~kg}$ i.a/ha, com $78,2 \%$ e $73,4 \%$, respectivamente, a- pós 30 e 45 dias da aplicação. Para a tiririca e O capim marmelada, o melhor controle foi obtido quando se aplicou metolachlor, em mistura com cyanazíne ou sozinho, com eficiência acima de $90 \%$ para tiririca e de $70 \%$ para capim-marmelada até 45 dias após aplicação Para as espécies não dominantes (mai oria dicotiled ôneas), o melh or controle foi obtido com a mistura de cyanazine + metolachlor na dose de $1,75+2,52 \mathrm{~kg}$ i.a/ha com $70,5 \%$ e $60,2 \%$, res pectiva mente, após 30 e 45 dias da aplicação. Para o to- tal das espécies, verificou-se que a mistura de cyanazyne + metolachlor, nas duas doses estudadas, foi que apresentou o melhor controle, com $66,2 \%$ e $67,3 \%$, respectivamente, após 30 dias e 63,3\% e 64,3\%, respectivamente, após 45 dias da aplicação.

Para a análise tecnológica da fibra, os dados obtidos podem ser observados no Quadro 5. Pela análise de variância, verificou-se que não houve nenhuma diferença significativa na maturação das fibras. Já para o comprimento, a mistura de cyanazyne + diuron $(0,8+0,8 \mathrm{~kg}$ i.a/ha $)$ apresentou o maior comprimento $(26,1 \mathrm{~mm})$ e cyanazine + metolacholor $(1,75+2,52 \mathrm{~kg}$ i.a / ha) o menor comprimento $(24,9 \mathrm{~mm})$, sem diferenças significativas com as testemunhas. Na uniformidade do comprimento os maiores valores foram obtidos com a mistura de cyanazine + metolachlor $(1,75+$ $2,52 \mathrm{~kg}$ i.a/ha) e diuron (1,6 $\mathrm{kg} \mathrm{i} \mathrm{a/} \mathrm{ha),} \mathrm{com}$ $47,2 \%$ e $46,9 \%$, respectivamente. No índice Micronaire, o maior valor foi obtido com diuron (1,6 kg i.a/ha), com 4,5 e o menor valor pela mistura de cyanazine + oxyzalin $(1,6+1,2 \mathrm{~kg}$ i.a/ha $)$, com 4,1. No índice Presley, as misturas de cyanazine e diuron e cyanazine + metolachlor nas duas doses estudadas, cyanazine + oryzalin na dose maior, assim como diuron e cyanazine isolados, apresentaram os mesmos valores.

\section{AGRA DECIMENTOS}

O autor agradece ao Técnico Agrícola Gisleno A. Drumond Pires, pela valiosa colaboração na condução deste trabalho, assim como à Shell Química S.A., pela colaboração financeira na realização do pres ente trabalho. 
Quadro 4. Percentagem de controle das plantas daninhas identificadas no ensaio de controle de plantas daninhas com cyanazine aplicado em mistura com outros herbicidas na cultura do algodão (Gossypium hirsutum L.) em Porteirinha, Norte de Minas. Ano Agrícola $1980 / 81$.

\begin{tabular}{|c|c|c|c|c|c|c|c|c|c|c|c|c|c|c|c|}
\hline Cyanazine + Diuron & $0,8+0,8$ & 0,0 & 20,0 & 24,9 & 29,7 & 33,6 & 34,8 & 78,8 & 69,7 & 11,8 & 11,7 & 0,0 & 0,0 & 0,0 & 0.0 \\
\hline Cyonazine + Diuron & $1,0+1,0$ & 0,0 & 50,0 & 73,6 & 70,5 & 48,2 & 40,5 & 68,2 & 59,8 & 0,0 & 0.0 & 44,8 & 40,0 & 56,0 & 53.6 \\
\hline Cyanazine + $0 r_{y} z a \ldots$ & $1,2+0,0$ & 0,0 & 47.0 & 76,8 & 60,8 & 0,0 & 0,0 & 54,1 & 45,1 & 26,5 & 35,0 & 48,6 & 29,4 & 51,0 & 47,9 \\
\hline Cyanazine + Orvzalin & $1.6+1.2$ & 71.4 & 79,4 & 77,4 & 75,6 & 44,5 & 46,8 & 18,8 & 12,3 & 61,8 & 60,0 & 56,2 & 50,3 & 60,8 & 58.2 \\
\hline $\begin{array}{c}\text { Cyanazine + Metola - } \\
\text { chlor }\end{array}$ & $1.4+2.0$ & 0,0 & 61,8 & 74,8 & 78,3 & 15,4 & 15,8 & 100,0 & 98,4 & 67,6 & 71,7 & 68,6 & 46.6 & 66,2 & 63.3 \\
\hline $\begin{array}{c}\text { Cyanazine + Metola - } \\
\text { chlor }\end{array}$ & $1,75+2,52$ & 0,0 & 73.5 & 70,4 & 71,0 & 32,7 & 21,5 & 98,8 & 95.1 & 76,5 & 70,0 & 70,5 & 50,2 & 67,3 & 64,3 \\
\hline Metolachlor & 2.52 & 0,0 & 11,8 & 80,9 & 76,3 & 0.0 & 0,0 & 100,0 & 97.5 & 61,8 & 70,0 & 37,1 & 26,1 & 31,8 & 42,2 \\
\hline Oryzalin & 1,12 & 71,4 & 82,4 & 75,9 & 71,4 & 0,0 & 0,0 & 55,3 & 49,2 & 8,8 & 40,0 & 9,1 & 0,0 & 45.9 & 42,4 \\
\hline Diuron & 1.6 & 0,0 & 17.6 & 72,8 & 72.1 & 50,0 & 50.6 & 88,3 & 82,2 & 47,0 & 58,3 & 5.7 & 0,0 & 57.0 & 55,2 \\
\hline Cyanazine & 1.75 & 0,0 & 61.8 & 39.7 & 38,6 & 73.4 & 76,5 & 76,5 & 68,0 & 58,8 & 56,7 & 0,0 & 0,0 & 26,4 & 32,4 \\
\hline $\begin{array}{l}\text { Testemunha } \\
\text { Capinada }\end{array}$ & - & 100,0 & 100,0 & 100,0 & 100,0 & 100,0 & 100,0 & 100,0 & 100.0 & 100,0 & 100,0 & 100,01 & 100,0 & 100,0 & 100,0 \\
\hline $\begin{array}{l}\text { Testemuna s/ } \\
\text { Capina (espécles/m }{ }^{2} \text { ) }\end{array}$ & - & 1.75 & 8.50 & 86,25 & 112,75 & 27.50 & 39,50 & 21,25 & 30,5 & 8,50 & 15,00 & 26,25 & 40,25 & 171,50 & 246,5 \\
\hline
\end{tabular}

Capina (espécies/m²

I/ Outras espécies: Eragrostis sp. (capim-fino), Caryophyllacea sp. (azedinho), Eclifta alba Hassk. (bolinha), Cenchrus echinatus L. (carrapicho de burro), Eragrotis sp. (capim-arroz), Digitaria sanguinalis L. (capim-colchão), Blainvillea biaris tata 0.C. (cai-cai). Desmanthus depressus (feijäozinho), Ipomoea sp. (jeti rana), Cassia tora L. (mata-pasto) Commelina sp. (macarrão), Bidens pilosa L. (picão), Spilanthes sp. (pimentinha), Richardia brasiliensis Gomez (quebra tigela). Cleone spinosa Jacq (umbunzinho), Sida sp. (malva-vermelha), Euphorbia hirta L. ( erva - de sangue). Mimosa sp. (malícia), e Gaya gracilipes i. Schum (malva). 
Quadro 5. Resultados médios das características tecnológicas da fibra no controle de plantas da ninhas com cyanazine, aplicado em mistura comoutros herbicidas na cultura do algodão (Gossypium hirsutum L.) em Porteirinha, Norte de Minas. Ano Agrícola $1980 /$ 81 .

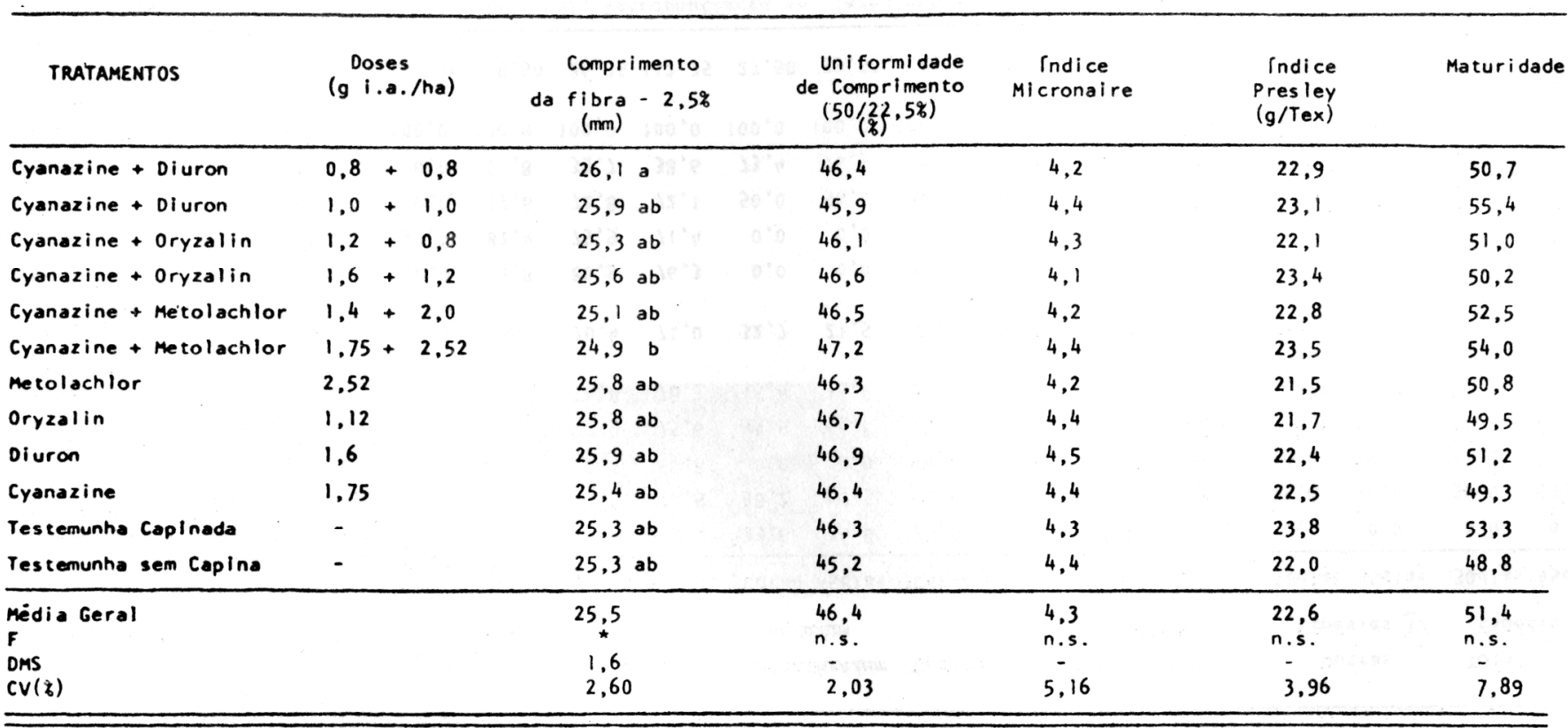

Obs.: Médias na mesma coluna, seguidas da mesma letra, não apresentam diferenças significativas no nivel de $5 \%$ de probabilidade pelo teste de Tukey. 


\section{LITERATURA CITADA}

1. Aguiar, J.C.; Alves, L.: Silva, G.; Beltrão, N. e Nóbrega, L.B. Competição de herbicidas na cultura do algodão herbáceo no Nordeste do Brasil. In: CONGRESSO BRASI LEIRO DE HERBICIDAS E ERVA $\bar{S}$ DANINHAS, 13., I1 héus/I tabuna, BA, 1980. Resumos. p.89.

2. Azevedo, J.J.; Chaves, R. \& Yepes, J.H. Mezclas de herbi cidas pré-emergentes para $e \bar{l}$ algodoeiro (Gossypium hirsutum L.) en el Valle del Cauca. In: SEMINĀRIO DE LA SOCIEDAD COLOMBIANA DE CONTROL DE MALEZAS Y FISIOLOGIA VEGE TAL (COMALFI), 3., Palmira, Colombia, 1971. Resumenes, p. 10-11.

3. Azevedo, D.M.P. de \& Beltrão, N.E. de M. Combinação de herbicidas no controle de plantas invasoras em algodo eiro herbáceo. In: CONGRESSO BRASILEIRO DE HERBICIDAS E ERVAS DANINHAS, 13., Ilhéus /Itabuna, BA, 1980. Resumos, p. 10 .

4. Blanco, H.G. \& Oliveira, D. A. Contribuição para determina ção do período de competição das plantas daninhas na cultura do algodoeiro (Gossy pium hirsutum L.). Biológico, 42(9-10): 201-5, 1976.

5. Laca-Buendia, J.P. de C.; Penna, J.C.V. \& Ferreira, M.B. Competição de herbicidas na cultura algodoeira (Gossypium hirsutum L.), no Triângulo Mineiro. In: SEMINARIO BRASILEIRO DE HERBICIDAS E ERVAS DANINHAS, 10., Santa Maria, RS, 1974. Resumos. p. 15-6.
6. Laca-Buendia, J.P. de C. \& Bar ros, A.T. de. Competição de misturas de herbicidas na cultura algodoeira (Gossypium hirsutum L.) nas principais regiões produtoras de Minas Gerais. In: SEMINARIO BRASILEIRO DE HERBICI DAS E ERVAS DANINHAS, 11, Londrina, PR, 1976. Resumos, $\mathrm{p} .42-3$.

7. Laca-Buendia, J.P. de C.; Purcino, A.A.C.; Ferreira, L. \& Ferreira, M.B. Competição de misturas de herbicidas nas principais regiões algodoeiras (Gossypium hirsutum L.) no Estado de Minas Gerais. Planta Daninha, 1(2): 5-16, 1978.

8. Laca-Buendia, J.P. de C.; Purcino, A.A.C.; Penna, J.C.V. \& Ferreira, L. Período crí tico de competição entre cō munidades de plantas daninhas e o algodoeiro (Gossypium hir sutum L. em M.G. Planta Danī nha, 2(2): 85-95, 1979.

9. Laca-Buendia, J.P. de C. Controle químico de plantas da ninhas na cultura al godoeira (Gossypium hirsutum L.) no Estado de Minas Gerais. In: SEMINARIO DE LA SOCIEDAD ECUATORIANA DE MALEZAS, 1., CONGRESSO SOCIEDAD LATINOAMERICALA DE MALEZASALAM, 5., Guayaquil, Ecuador, 1980. Resumenes, p. 29.

10. Saldarriaga, A.; Cruz, de La, R. \& Lagos, E. Estudio de herbicidas en algodón. In: SEMINARIO DE LA SOCIEDAD COLOMBIANA DE CONTROL DE MA

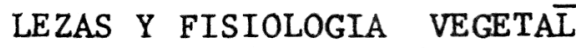
(COMALFI), 1. Bogotá, Colombia, 1969. Resumenes, p. 17-19. 
11. Silva, R.J.M. da; Macedo,A.M. ; Cerqueira, W.P. \& Morais, J. de D. Efeito da combina ção de herbicidas no contrö le de ervas daninhas e ná produção do algodoeiro herbáceo em Goiás. EMGOBA, 1981, 12p. (Boletim Técnico n? 4).

12. Torrado, G.E. \& Gardenas, J. Mezclas de herbicidas y nue vos productos para el control de malezas en algodón. In: SEMINARIO DE LA SOCIEDAD COLOMBIANA DF CON
TROL DE MALEZAS Y FISIOLO GIA VEGETAL (COMALFI), 3., Palmira, Colombia, 1971. Re sumenes, p.10-11

13. Yepes, J.M. \& Chaves, R. Herbi cidas pré-emergentes en trá tamento de presiembra incorporado para el al godonero. In: SEMINARIO DE LA SOCIEDAD COLOMBIANA DE CONTROL DE MALEZAS Y FISIOLO GIA VEGETAL (COMALFI). 2., Bogotá, Colombia, 1980.

Resumenes, p.42-49. 